\title{
BMJ Open Randomised controlled trial of a web- based programme in sustaining best practice alcohol management practices at community sports clubs: a study protocol
}

\author{
Tameka McFadyen, ${ }^{1,2}$ Luke Wolfenden, ${ }^{1,2}$ Melanie Kingsland, ${ }^{1}$ Jennifer Tindall, ${ }^{2}$ \\ Bosco Rowland, ${ }^{3}$ Shauna Sherker, ${ }^{4}$ Karen Gillham, ${ }^{2}$ Rachael Heaton, ${ }^{4}$ \\ Tara Clinton-McHarg, ${ }^{1}$ Christophe Lecathelinais, ${ }^{2}$ Daisy Brooke,${ }^{4}$ John Wiggers ${ }^{1,2}$
}

To cite: McFadyen T, Wolfenden L, Kingsland M, et al. Randomised controlled trial of a web-based programme in sustaining best practice alcohol management practices at community sports clubs: a study protocol. BMJ Open 2018;8:e017796. doi:10.1136/ bmjopen-2017-017796

- Prepublication history for this paper is available online. To view these files, please visit the journal online (http://dx.doi. org/10.1136/bmjopen-2017017796).

Received 17 May 2017 Revised 1 December 2017 Accepted 6 December 2017

\section{Check for updates}

${ }^{1}$ School of Medicine and Public Health, The University of Newcastle, Callaghan, New South Wales, Australia

${ }^{2}$ Population Health, Hunter New England Health, Wallsend, New South Wales, Australia

${ }^{3}$ School of Psychology, Deakin University, Burwood, Victoria,

Australia

${ }^{4}$ Good Sports, Alcohol and Drug Foundation, Melbourne, Victoria, Australia

Correspondence to Tameka McFadyen; tameka-rae.small@hnehealth. nsw.gov.au

\section{ABSTRACT}

Introduction Community-based interventions have been found to effectively increase the implementation of alcohol management practices and reduce excessive alcohol use and alcohol-related harm at sports clubs. However, once implementation support ceases there may be a reduction in such intervention effects. Thus, ongoing contribution to improving the health of the community is diminished; sustaining practice implementation is a key determinant to address this. One possible solution to the strategic and logistical challenges of sustainability involves the use of the web. The primary aim of this study is to assess the effectiveness of a web-based programme in sustaining the implementation of alcohol management practices by community football clubs. The secondary aim is to assess the effectiveness of the programme in preventing excessive alcohol consumption and alcohol-related harm among members of community football clubs.

Methods and analysis The study will employ a repeat randomised controlled trial design and be conducted in regional and metropolitan areas within two states of Australia. Community level football clubs who are currently accredited with an existing alcohol management programme ('Good Sports') and implementing at least 10 of the 13 core alcohol management practices (eg, not serving alcohol to $<18$-year-olds) required by the programme will be recruited and randomised to either a web-based sustainability programme or a 'minimal contact' programme. The primary outcome measures are the proportion of football clubs implementing $\geq 10$ of the 13 required alcohol management practices and the mean number of those practices being implemented at 3-year follow-up. Secondary outcomes include: the proportion of club members who report risky drinking at their club, the Alcohol Use Disorder Identification Test (AUDIT) score and mean AUDIT score of club members. Outcome data will be collected via observation at the club during a 1-day visit to a home game, conducted by trained research assistants at baseline and follow-up.

\section{Strengths and limitations of this study}

- The study has a strong design incorporating random allocation, blinding of research personnel undertaking data collection and the use of objective outcome measures.

- The intervention is a multifaceted novel design, based on theoretical frameworks and evidence from past research in this area.

- The study will be conducted with community football clubs only, therefore the generalisation of outcomes from this study will be uncertain for other sporting codes and community settings generally.

Ethics and dissemination The study was approved by The University of Newcastle Human Research Ethics Committee (reference: H-2013-0429). Study findings will be disseminated widely through peer-reviewed publications and conference presentations.

Trial registration number ACTRN12614000746639; Preresults.

\section{INTRODUCTION}

Excessive use of alcohol is responsible for more than 3 million deaths and over 200 varieties of disease and injuries worldwide each year. ${ }^{1}$ One population group that has been identified as consuming excessive amounts of alcohol are those involved with organised sport (both players and supporters), including those at the non-elite/community level. ${ }^{2-7}$ Young men, particularly those involved in contact team sports, have been reported to have a high prevalence of excessive alcohol consumption. ${ }^{358}$ Additionally, when compared with people not associated with sport, a greater prevalence of alcohol-related harm has been reported among players and spectators of a range of sports, 
again, particularly male dominated, team and contact sports. ${ }^{79-11}$ Alcohol-related harm refers to both the immediate and log-term negative consequences associated with excessive alcohol consumption, such as injury, assaults, accidents and at home abuse, as well as chronic health conditions and some cases of suicide. ${ }^{1}$

Community sports clubs have been cited as an opportune setting to address excessive alcohol use and alcohol-related harm among sportspeople and fans. ${ }^{12}$ Evidence from a $2 \frac{1}{2}$ year multistrategic randomised controlled trial of an alcohol management programme (the 'Good Sport' programme) conducted in community sports clubs in Australia found a significant increase in the implementation of alcohol management practices in intervention clubs (38\%) compared with control clubs $(25 \%) .{ }^{13}$ The trial also found a significant postintervention difference in the proportion of intervention club members who engaged in risky alcohol consumption at the club (19\%) and in the proportion of members at risk of alcohol-related harm (38\%), compared with control club members (risky drinking: 24\%; risk of alcohol-related harm: $45 \%) .{ }^{14}$ The intervention included multiple strategies to support clubs in implementing alcohol management practices, including face-to-face and telephone-based support from a dedicated project officer.

The sustainability of improved practice implementation after initial intervention is a key determinant of whether effective programmes can make an ongoing contribution to improving the health of the community. ${ }^{15}{ }^{16}$ However, sustained implementation of effective health promotion programmes is a common challenge across numerous community settings. ${ }^{15}$ A review of 17 studies of a variety of community-based health-related programmes in the USA and Canada found that only 29\% ( $\mathrm{n}=5)$ achieved sustainability of implementation of at least one programme component postintervention. ${ }^{17}$ No studies conducted within the sports club setting have reported on the sustainability of alcohol management practices. Given the suggested challenges of sustaining programme implementation, particularly when programmes are implemented at scale across large populations and geographic areas, effective and efficient mechanisms for achieving sustained programme implementation and benefit are required. ${ }^{18}$ This is most likely true for the community sport setting specifically and by service provider organisations more generally.

One possible solution to the strategic and logistical challenges of programme sustainability in sports and other settings involves the use of web-based programmes to sustain the implementation of programme elements. Web-based programmes have the potential to be delivered at relatively low cost to large numbers of sites across large geographic areas. While web-based programmes have been used to support both implementation and quality improvement initiatives in other settings, such as hospitals, general healthcare ${ }^{1920}$ and schools, ${ }^{21-23}$ there has not been any reports of rigorous evaluations of their effectiveness in maintaining sporting clubs' adherence to alcohol management practices. Given this, a study will be conducted with the primary aim of assessing the effectiveness of a web-based programme in sustaining the implementation of best practice alcohol management practices by community football clubs. The secondary aim is to assess the effectiveness of the programme in preventing excessive alcohol consumption and alcohol-related harm among members of community football clubs.

\section{METHODS AND ANALYSIS \\ Study design}

The study will employ a repeat cross-sectional randomised controlled trial design (see figure 1). Clubs will either be randomised into a 'web-based sustainability program' or an 'ethical minimal contact' control group.

\section{Research setting}

The research will occur in Australia, in regional areas of the state of New South Wales (NSW) and throughout metropolitan and regional areas of the state of Victoria.

\section{Participants and research eligibility \\ Football clubs}

Non-elite, community-level clubs that currently hold the highest level of accreditation in an alcohol harm-reduction programme ('Good Sports') ${ }^{24}$ will be recruited to participate in the trial. Sporting clubs interested in becoming involved in the Good Sports programme, a free nationwide programme, register their details and are required to implement alcohol management practices of increasing comprehensiveness across three levels of accreditation. Level 3 is the highest level of accreditation with the programme. There are currently approximately 2600 level 3 Good Sports clubs in Australia. All clubs participating in this trial will have been involved with the Good Sports programme for a minimum of 5 years to progress to the highest level (level 3) of accreditation.

Football clubs will be eligible to participate in the study if they: have held the highest level of accreditation (level 3) within the Good Sports programme for a minimum of 12 months; adhere to the alcohol management practices required of level 3 Good Sports clubs; are a non-elite community-level football club; are an Australian Football League, Rugby League, Rugby Union or Soccer club; hold a current valid liquor licence; currently sell alcohol; have at least one senior (over 18s) team; and report having access to the internet.

The football codes that are eligible to participate in this study are among the most popular organised sports in Australia $^{25}$ and people involved with these sports as players or spectators have been identified as particularly at risk of alcohol-related harm relative to other sporting codes.

\section{Football club members}

Club members will be eligible to participate in the study if they are: current members/affiliates of a participating club (including players, committee members, regular 


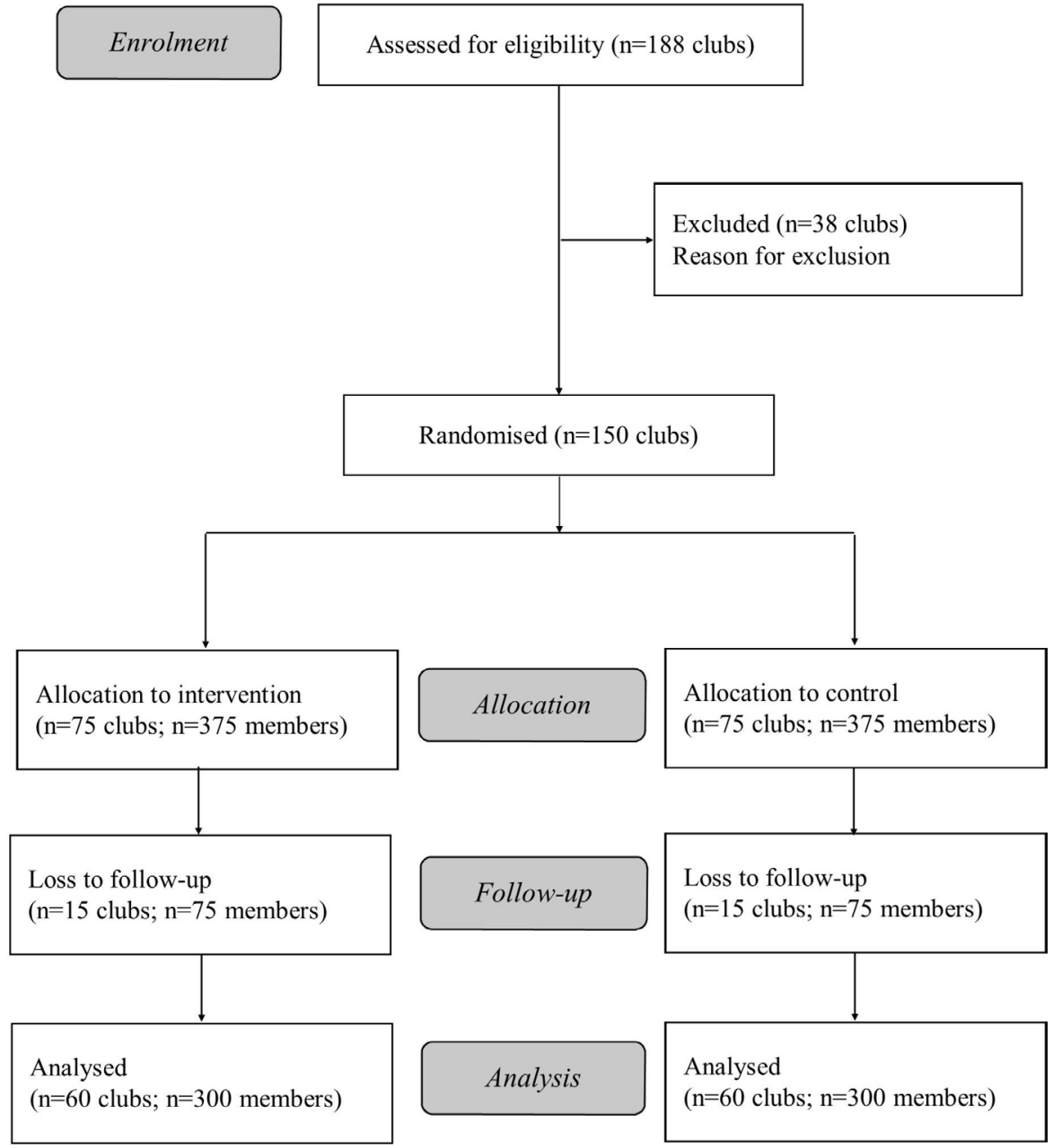

Figure 1 Consort flow chart estimating the possible progress of participants through the trial from enrolment to final analysis.

spectators/fans and coaches), at least 18 years of age and speak English.

\section{Recruitment procedures}

Football clubs

A list of level 3 Good Sports football clubs within the study areas will be generated by the research team from programme records. A club representative (eg, club president, vice president or secretary) from eligible clubs will be sent a study information sheet inviting the club to participate in the study. Two weeks after sending the letter, club representatives will be contacted via telephone to screen the club for eligibility based on the above criteria and assess interest in study participation. If club representatives do not recall receiving the study information sheet, contact details will be confirmed and an additional information sheet will be sent to them either via email or postal mail. Follow-up phone calls will occur until club representatives are able to make informed decisions about their club's participation in the study. It is envisaged that some clubs may require this decision to be taken to the club management committee. If club representatives are not able to be contacted, alternative contacts will be sought via programme databases, relevant football code association websites and other publicly available forums. Consenting clubs will be asked to complete and sign a consent form and return it to the research team. All recruitment procedures will be managed by a dedicated member of the research team.

Football club members

At baseline and follow-up a separate independent sample of club members (players, committee members, regular spectators/fans and coaches) will be recruited at club grounds during a senior home game using a pilot-tested, standardised recruitment protocol which will detail specified locations for recruitment within the club grounds (ie, club bar or canteen). A random number sequence will be generated by a computer program and integrated into the data collection tool. The random number sequence will dictate the order in which members at the grounds are to be approached by the research assistants. These randomly selected members will then be screened for eligibility, and those who are eligible will be provided with a study information sheet and invited to participate in the study. Eligible and consenting club members will be asked to provide their contact details to the research staff. This recruitment process will continue until a maximum of 20 members' contact details are collected at each club/game. The 20 club members who agreed to provide contact details will receive a phone call from the research team formally 
inviting them to participate in the study. This process will occur until five members from each participating club have been enrolled into the study.

For recruitment of both clubs and members, strategies previously found to maximise research participation will be used, including prenotification of the study and opportunity to participate, direct telephone contact with participants to invite participation, multiple contact attempts, access to research staff for further clarification regarding participation and the use of dedicated, specifically trained research staff to undertake the recruitment process. Such recruitment strategies have been previously used successfully by the research team. ${ }^{26-28}$

\section{RANDOM ALLOCATION OF SPORTS CLUBS}

Clubs will be randomly allocated (after baseline data collection) in a 1:1 ratio to either the intervention or control group by a statistician independent to the research team using a computerised random number function. The randomisation procedure will be stratified by sports code. Research conducted by the research team $^{14}$ and others ${ }^{29}$ has previously demonstrated an association between this factor and secondary outcomes related to excessive alcohol consumption and alcohol-related harm. Additionally, due to operational differences between football clubs, the randomisation procedure will also be stratified by state (NSW or Victoria).

\section{BLINDING}

Research assistants conducting the field observations for the outcome measures will be blind to group allocation and not be involved in any other aspect of the trial. Effectiveness of research personnel blinding will be tested by asking research assistants to nominate group allocation of clubs following collection of postintervention data. Additionally, the analysis of primary trial outcomes will be undertaken by a statistician who will be blind to study group allocation through use of and dummy variable for allocation. Due to the difficulty in blinding clubs to group allocation, this will be an open trial with club representatives being told of the treatment status of their club following pretest data collection.

\section{INTERVENTION}

\section{Alcohol management practices}

Intervention clubs will be supported to maintain the implementation of the alcohol management practices outlined in box via a web-based programme. Each intervention club will be assigned a unique identifier so the research team can tract their progress through, and engagement with the programme. The alcohol management practices are consistent with legislation and guidelines regarding the sale and supply of alcohol in licensed premises $^{30-32}$ and have been found to be associated with
Box Alcohol management practices

Practice

Substantial food provided when alcohol sold. ${ }^{56}$

Non-alcoholic drinks $10 \%$ cheaper than full-strength alcoholic drinks. $^{32-34}$

Drunk/intoxicated people not allowed to enter club. ${ }^{35} 3657$

Low-alcoholic drinks 10\% cheaper than full-strength alcoholic drinks. $^{32} 53$ 58-60

Four non-alcoholic options available for purchase. ${ }^{32} 5361$

People under 18 years of age do not serve alcohol. ${ }^{57}$

Drunk/intoxicated people not served alcohol. ${ }^{35} 3657$

Drunk/intoxicated people not permitted to remain on club premises. ${ }^{57}$

Free water provided when alcohol sold. ${ }^{53}$

Staff do not consume alcohol while on duty. ${ }^{35} 36$

One low-alcoholic drink option available. ${ }^{32} 5361$

Licensing signs visible at all bars. ${ }^{3561}$

No drink promotions undertaken at the club (happy hour, all-you-can-

drink functions, alcohol-only awards and prizes, cheap drinks, drinking games, drinking vouchers/cards). ${ }^{32} 3536566263$

lower levels of alcohol consumption in licensed premises, broadly, ${ }^{33} 34$ and community sports clubs, specifically. ${ }^{35-37}$

\section{Web-based sustainability programme}

A web-based approach was selected given the capacity for web-based support to be: (1) efficiently delivered to a large number of sports clubs located in a wide geographic area; (2) maintained and updated centrally at relatively low cost; (3) tailored to the needs of individual clubs. Such computer-based interventions have been used to improve and sustain the health-promoting practices of organisations generally. ${ }^{38}$ Preliminary unpublished data (2014) collected by the research team suggest that a web-based intervention will be acceptable to sports club representatives as they reported a high level of use of computers and web-based programmes $(n=202), 81 \%$ reported that club representative tasks were mainly undertaken on computer-based programmes, $62 \%$ reported using a web-based programme for the majority of club tasks and 99\% indicated that they had a website or social media page.

An expert advisory group consisting of community sports club representatives, health promotion practitioners and experts in community organisational change and reducing alcohol-related harm associated with licensed premises will develop the programme based on theory ${ }^{39} 40$ and evidence. ${ }^{13}$ The advisory group will continue to meet via the telephone and face-to-face and undertake programme testing to develop the web-based programme. The web-based programme will only be available to those clubs allocated to the intervention group of the trial, this isnot a publicly available web site. It can only be accessed by a personalised login number.

\section{Conceptual model and strategies}

A frequently identified limitation of sustainability research has been the lack of conceptual models to guide intervention development. ${ }^{1841}$ To address this, the web-based sustainability programme will be developed based on the 
Sustainability Framework, ${ }^{39}$ which identifies the following domains as being important in sustaining the delivery of programme practices:

- Environmental: having a supportive internal and external climate.

- Organisational capacity: having internal support and resources needed to effectively manage the programme.

- Programme adaptation: taking actions that adapt the programme to ensure its ongoing effectiveness.

- Communications: having strategic communication with stakeholders and the public about the programme.

- Strategic planning: using processes that guide the programme's directions, goals and strategies.

- Programme evaluation: assessing the programme to inform planning and document results.

- Partnership: having connections between the programme and its stakeholders.

- Funding stability: having a consistent financial base for the programme.

Additionally, as the programme will be web based, the Persuasive Systems Design framework ${ }^{40}$ will be used to ensure programme usability. This framework recognises the importance of the following elements that are relevant to the proposed programme:

- Reduction: system reduces complex behaviour into simple tasks.

- Tunnelling: using the system to guide users through a process or experience provides opportunities to persuade along the way.

- Tailoring: information tailored to the potential needs, interests, personality, usage context or other factors relevant to a user group.

- Personalisation: personalised content.

- Self-monitoring: keeps track of one's own performance or status, supports the user in achieving goals.

- Simulation: provides simulations, links between cause and effect.

- Praise: make users more open to persuasion.

- Rewards: reward targeted behaviour.

- Reminders: remind users of the targeted behaviour.

- Suggestion: offering fitting suggestion for persuasion.

The use of such frameworks in the design of practice change initiatives, particularly those operating at multiple levels of complex systems, has been suggested to be important in facilitating effective practice change. ${ }^{42}$

The programme will include the following functions:

1. Annual online assessment. At the start of each year (sporting season), the club president or a nominated representative from each intervention club (eg, club secretary) will complete an online monitoring assessment. The monitoring assessment will require the club representative to complete a series of questions regarding their club's alcohol management practices (box). The completion of the monitoring report will highlight any practices that clubs are not undertaking.
The responses to the annual assessment will be sent to each club committee.

2. Action plan. An action plan, individualised for each club, will be generated from the identified incomplete alcohol management practices from the monitoring report. It will contain specific information about such practices, such as why the practice is important, if it is required by legislation and steps to effectively implement the practice.

3. Tools and resources. From the action plan, clubs will be guided to practise specific tools and resources to support clubs in implementing identified incomplete alcohol management practices. All intervention clubs will have access to all tools and resources via the online intervention programme.

\section{Programme implementation strategies}

The programme will be designed to include implementation strategies (table 1) that address common impediments to organisations sustaining new initiatives or innovations and impediments that are particularly relevant to the volunteer community sport club setting such as high staff turnover, limited resources, lack of time and competing demands. ${ }^{43-45}$

Table 1 outlines the programme implementation strategies mapped against the domains of the Sustainability Framework and the Persuasive Systems Design framework.

\section{Programme delivery}

Programme delivery will occur over three successive Australian winter sporting seasons (April to September 2015-2017). Participating intervention clubs will receive contact from a member of the programme team a maximum of four times via telephone and a maximum of four times via email during each intervention season. Programme implementation completion will occur at the end of the 3-year intervention period with clubs having completed three rounds of annual online assessments and action planning.

\section{Programme quality assurance}

User acceptance testing will be undertaken with a sample of representatives from community sporting clubs. Web-based programme testing will occur, with feedback being integrated into the final version of the programme. Ongoing quality assurance checks will be undertaken as clubs engage with the web-based programme. Research staff will perform annual checks on all links to external sites within the intervention site to ensure they remain current, and club logins will be monitored to ensure no barriers to intervention access, such as site blocking or browser issues.

\section{CONTROL GROUP CLUBS}

Control group clubs will not have access to the web-based programme or any of the intervention programme resources. Control group clubs will receive minimal 
Table 1 Intervention implementation strategies and conceptual frameworks

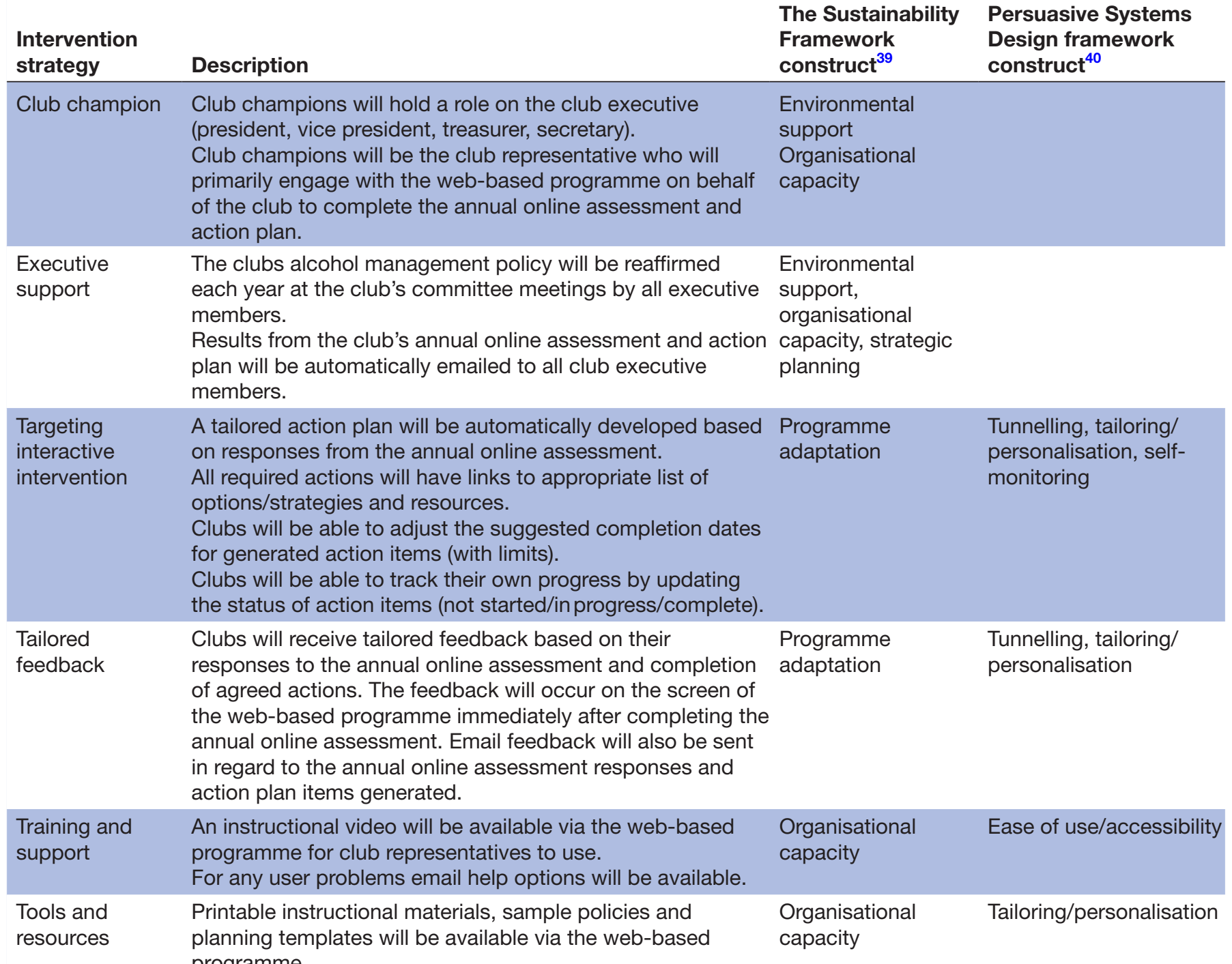
programme.

\begin{tabular}{|c|c|c|c|}
\hline $\begin{array}{l}\text { Systems and } \\
\text { prompts }\end{array}$ & $\begin{array}{l}\text { Email reminders will be automatically sent to prompt annual } \\
\text { online assessment completion and when action plan } \\
\text { items' due dates are approaching or are overdue. }\end{array}$ & Environmental & $\begin{array}{l}\text { Reminders and prompts, } \\
\text { tailoring/personalisation }\end{array}$ \\
\hline $\begin{array}{l}\text { Communication } \\
\text { and marketing }\end{array}$ & $\begin{array}{l}\text { An independent nominee (a community stakeholder) will } \\
\text { be sent a letter from the programme informing them of the } \\
\text { club's progress. }\end{array}$ & $\begin{array}{l}\text { Communication, } \\
\text { partnership }\end{array}$ & $\begin{array}{l}\text { Praise, rewards and } \\
\text { recognition }\end{array}$ \\
\hline $\begin{array}{l}\text { Recognition and } \\
\text { reward }\end{array}$ & $\begin{array}{l}\text { Automatic notification and praise via emails will be sent to } \\
\text { club champions and club executives when the annual online } \\
\text { assessment and action plan is complete. } \\
\text { 'Good News' stories of clubs progressing through the } \\
\text { programme will be positioned on the login screen. }\end{array}$ & & $\begin{array}{l}\text { Praise, rewards and } \\
\text { recognition }\end{array}$ \\
\hline
\end{tabular}

programme support throughout the intervention period. This will entail one phone contact occasion initiated by the research team during the 3-year intervention period. Additionally, the research team will provide reactive support, on an as-needs basis in instances where a club seeks support about an alcohol-related incident or concern. The research team will assess each support request to ensure group contamination does not occur and record any additional contact made with clubs.

\section{MEASURES}

Primary outcomes

1. The proportion of clubs implementing $\geq 10$ of the 13 required alcohol management practices (box) at follow-up.

2. The mean number of alcohol management practices being implemented at follow-up.

Such implementation is consistent with research ${ }^{4647}$ that recognises that the implementation of multiple strategies 
targeting different aspects of alcohol management (availability, promotion and alcohol service) is more successful in harm minimisation than the implementation of individual strategies. ${ }^{32} 48$

\section{Secondary outcomes}

1. The proportion of clubs implementing each of the 13 alcohol management practices.

2. The proportion of club members who report drinking alcohol at risky levels at sporting clubs. This outcome is measured by the graduated frequency index, ${ }^{49}$ with risky drinking defined as five or more standard drinks of alcohol on one drinking occasion ${ }^{50}$ at least once a month at the club.

3 . The proportion of club members at risk of alcohol-related harm, as measured by a total Alcohol Use Disorder Identification Test (AUDIT) score of $\geq 8 .{ }^{51}$

4. The mean AUDIT score of club members.

\section{Process data}

Process data measures will be collected for each intervention club and will include: total time to complete annual report and action plan online, number of logins to complete annual report and action plan, number of tools and resources accessed/downloaded and average number of action items generated.

\section{Club characteristics}

The following data will be collected on participating sports clubs: number of senior (18years of age and over) and junior (under 18 years of age) teams and members registered with the club, football code and postcode of sporting club.

\section{DATA COLLECTION PROCEDURES Primary outcome}

Baseline and follow-up data for the primary trial outcome will be collected by covert field observation of club practices by trained research assistants. Direct observation represents the gold standard when measuring complex environments and organisational behaviours. ${ }^{52}$ Observations will occur during a home game at a participating club ground. Participating clubs will not be aware of the exact day that the observation will occur. Given that many clubs have multiple teams, the observation will occur during the most senior game. Observation staff will be trained by the research team, and will be required to complete examples of alcohol practice observation scenarios to a satisfactory level. Observers will assess each of the alcohol management practices outlined in box during the field observation. Protocols and methods for such observations have been developed and successfully implemented by the research team during more than 200 observations of sports clubs, hotels and nightclubs as part of previous trials, ${ }^{53}$ and pilot testing will be conducted with four clubs prior to commencement of data collection to refine the tool.

\section{Secondary outcome}

Secondary data collection will occur via a Computer Assisted Telephone Interview with club members recruited from the field observations at baseline (2015) and follow-up (2017).

\section{Process data}

Measures outlined above will be collected via reports generated through the web-based software and Google Analytics. The research team will run reports at the end of the intervention period.

\section{Club characteristic data}

The operational characteristics of clubs, demographics and attitudes of sports club representatives will be collected during a telephone survey with the club representative.

\section{Data management}

Data management will primarily be the responsibility of a statistician, independent of the research team and trial activities. Management of trial data will be in accordance with a data management protocol, which will be developed for approval by the Project Advisory Group. As a requirement of ethics approval, all data collected for the trial will be securely stored, accessible only to primary researchers and statisticians through the allocation of access rights. Confidential participant data including contact details (eg, phone numbers) will be stored in a secure data set that is not linked to survey response data sets. An independent statistician will be the only person with access to confidential participant data.

\section{SAMPLE SIZE AND POWER CALCULATIONS}

Assuming at follow-up that $70 \%$ of clubs in the control group have maintained $\geq 10$ of the 13 required alcohol management practices (box) (ie, the prevalence of adequate practice implementation in the control group falls from $100 \%$ at baseline to $70 \%$ at follow-up), a sample size of 60 clubs per group will be sufficient to detect an absolute difference of $20 \%$ between groups at follow-up (which assumes that the prevalence of clubs in the intervention group that maintain $80 \%$ of required practices falls from $100 \%$ at baseline to $90 \%$ at follow-up) with $80 \%$ power and alpha of 0.05 .

\section{Statistical analysis}

\section{Primary outcome}

The primary trial outcomes will be assessed by examining group differences at follow-up in the (1) prevalence of clubs maintaining $\geq 10$ of the 13 required alcohol management and (2) the mean number of practices being implemented by clubs. Analysis will be performed in SAS V.9.3 statistical software and under an intention-to-treat approach. Dichotomous primary outcomes will be assessed using logistic regression models. Continuous primary outcomes will be assessed using linear regression models. All models will be controlled for baseline outcome values. Sensitivity 
analysis will be performed to test a range of assumptions for missing data at follow-up as recommended by White $e t a l .{ }^{55}$ Statistical tests will use an alpha of 0.05 .

\section{Research trial coordination}

The trial will be overseen by a Project Advisory Group, which is chaired by one of the chief investigators of the Australia Research Council grant. The group includes representatives from The University of Newcastle, Hunter New England Population Health, the Alcohol and Drug Foundation and Deakin University.

A project team has also been formed to coordinate the implementation of the trial in accordance with the trial protocol. This team consists of staff members of The University of Newcastle, Hunter New England Population Health and the Alcohol and Drug Foundation. Data management is the responsibility of a Hunter New England Population Health statistician, otherwise independent of the research team and trial activities. Management of trial data is in accordance with a data management protocol, developed and approved by the Project Advisory Group.

\section{Trial discontinuation or modification}

There are no predetermined criteria for discontinuing or modifying the trial. While unintended adverse events to trial participants, researchers or other community members are not anticipated, any such events will be forwarded to The University of Newcastle Human Research Ethics Committee (HREC) in accordance with the conditions of ethics approval. Should the research team or HREC consider it appropriate, the trial protocol or procedures may be modified to prevent such harm. Any protocol modification will be communicated through modification of the trial registration listed in the Australian New Zealand Clinical Trials Registry and through publications disseminating trial results.

\section{DISCUSSION}

There is an absence of research evidence with regard to how to sustain alcohol management practices in the sporting club setting. This will be the first randomised controlled trial to measure the effectiveness of a web-based programme in sustaining such practices, in not just the community football club setting but any community setting. The study has a strong design that incorporates computerised random allocation, blinding of data collection personnel and observational data collection methods. In addition, the intervention is based on a strong theoretical framework and research evidence from the sports and other settings. This study will provide clubs in regional and remote geographical locations, who previously had restricted face-to-face programme support, extensive access to web-based support for the implementation of alcohol management practices. The use of such web-based support may be applicable to other community settings which have wide geographical spread of sites, such as schools and childcare centres. The findings from this study will provide a basis for further research in the field and provide potentially important findings on programme sustainability for both policymakers and those providing health promotion programmes to community sporting groups and community organisations more broadly.

Contributors TM, LW, MK, JW, JT, KG, BR, SS and RH contributed to the study method design and intervention development. CL, LW, MK, JW and TM contributed to analysis design. TM, LW, MK, JW, JT, KG, BR, SS, RH, TCMH and DB contributed to manuscript development and review.

Funding This work is supported by the Australian Research Council (LP130100851), Hunter New England Population Health and the Alcohol and Drug Foundation.

Competing interests None declared.

Patient consent Obtained.

Ethics approval The University of Newcastle Human Research Ethics Committee. Provenance and peer review Not commissioned; externally peer reviewed.

Open Access This is an Open Access article distributed in accordance with the Creative Commons Attribution Non Commercial (CC BY-NC 4.0) license, which permits others to distribute, remix, adapt, build upon this work non-commercially, and license their derivative works on different terms, provided the original work is properly cited and the use is non-commercial. See: http://creativecommons.org/ licenses/by-nc/4.0/

(C) Article author(s) (or their employer(s) unless otherwise stated in the text of the article) 2018. All rights reserved. No commercial use is permitted unless otherwise expressly granted.

\section{REFERENCES}

1. World Health Organisation. Global status report on alcohol and health 2014. Geneva: World Health Organisation, 2014.

2. Poortinga W. Associations of physical activity with smoking and alcohol consumption: a sport or occupation effect? Prev Med 2007;45:66-70.

3. Brenner J, Swanik K. High-risk drinking characteristics in collegiate athletes. J Am Coll Health 2007;56:267-72.

4. Nelson TF, Wechsler H. Alcohol and college athletes. Med Sci Sports Exerc 2001:33:43-7.

5. Snow P, Munro G. Alcohol consumption in amateur Australian rules football clubs: evidence from a rural region. Health Promot $J$ Austr 2000;10:237-43.

6. O' Brien KS, Blackie JM, Hunter JA. Hazardous drinking in elite New Zealand sportspeople. Alcohol Alcohol 2005;40:239-41.

7. O'Farrell AM, Allwright SP, Kenny SC, et al. Alcohol use among amateur sportsmen in Ireland. BMC Res Notes 2010;3:313.

8. Martha C, Grélot L, Peretti-Watel P. Participants' sports characteristics related to heavy episodic drinking among French students. Int J Drug Policy 2009;20:152-60.

9. Merlo LJ, Ahmedani BK, Barondess DA, et al. Alcohol consumption associated with collegiate American football pre-game festivities. Drug Alcohol Depend 2011;116:242-5.

10. Lawson JS, Evans AR. Prodigious alcohol consumption by Australian rugby league footballers. Drug Alcohol Rev 1992;11:193-5.

11. Bedendo A, Opaleye ES, Andrade AL, et al. Heavy episodic drinking and soccer practice among high school students in Brazil: the contextual aspects of this relationship. BMC Public Health 2013:13:247.

12. Mentha R, Wakerman J. An evaluation of the Australian Football League Central Australian responsible alcohol strategy 2005-07. Health Promot J Austr 2009;20:208-13.

13. Kingsland M, Wolfenden L, Tindall J, et al. Improving the implementation of responsible alcohol management practices by community sporting clubs: A randomised controlled trial. Drug Alcohol Rev 2015;34:447-57.

14. Kingsland $\mathrm{M}$, Wolfenden $\mathrm{L}$, Tindall J, et al. Tackling risky alcohol consumption in sport: a cluster randomised controlled trial of an 
alcohol management intervention with community football clubs. $J$ Epidemiol Community Health 2015;69.

15. Shediac-Rizkallah MC, Bone LR. Planning for the sustainability of community-based health programs: conceptual frameworks and future directions for research, practice and policy. Health Educ Res 1998;13:87-108.

16. Oldenburg BF, Ffrench ML, Sallis JF. Health behavior research: the quality of the evidence base. Am J Health Promot 2000;14:253-7.

17. Scheirer MA. Is sustainability possible? A review and commentary on empirical studies of program sustainability. Am J Eval 2005;26:320-47.

18. Milat AJ, Newson R, King L, et al. A guide to scaling up population health interventions. Public Health Res Pract 2016;26:e2611604.

19. Robertson A, Cresswell K, Takian A, et al. Implementation and adoption of nationwide electronic health records in secondary care in England: qualitative analysis of interim results from a prospective national evaluation. BMJ 2010;341:c4564.

20. Roshanov PS, Misra S, Gerstein HC, et al. Computerized clinical decision support systems for chronic disease management: a decision-maker-researcher partnership systematic review. Implement Sci 2011;6:92.

21. Hamel LM, Robbins LB, Wilbur J. Computer- and web-based interventions to increase preadolescent and adolescent physical activity: a systematic review. J Adv Nurs 2011;67:251-68.

22. Chen JL, Wilkosz ME. Efficacy of technology-based interventions for obesity prevention in adolescents: a systematic review. Adolesc Health Med Ther 2014;5:159-70.

23. Ezendam NP, Brug J, Oenema A. Evaluation of the Web-based computer-tailored FATaintPHAT intervention to promote energy balance among adolescents: results from a school cluster randomized trial. Arch Pediatr Adolesc Med 2012;166:248-55.

24. Good Sports. Alcohol and drug foundation. http://goodsports.com. au/

25. Australian Bureau of Statistics. Perspectives on sport, May 2009: feature article 1: football: four games, one name. Canberra: Australian Bureau of Statistics, 2009.

26. Mail DD. Internet surveys: the tailored design method. New York: Wiley, 2000.

27. Edwards $\mathrm{P}$, Roberts I, Clarke $\mathrm{M}$, et al. Increasing response rates to postal questionnaires: systematic review. BMJ 2002;324:1183.

28. Wolfenden L, Kypri K, Freund M, et al. Obtaining active parental consent for school-based research: a guide for researchers. Aust NZ $J$ Public Health 2009;33:270-5.

29. Fone DL, Farewell DM, White J, et al. Socioeconomic patterning of excess alcohol consumption and binge drinking: a cross-sectional study of multilevel associations with neighbourhood deprivation. BMJ Open 2013;3:e002337.

30. New South Wales Government. Liquor Act 2007. 2007 http://www. austlii.edu.au/au/legis/nsw/consol_act/la2007107/ (accessed Jan 2017)

31. Victoria. Liquor Control Reform Act 1998. 1998 http://www.austlii. edu.au/au/legis/vic/consol_act//cra1998266/ (accessed Jan 2017).

32. Babor T. Alcohol: no ordinary commodity: research and public policy: Oxford University Press, 2010.

33. Chikritzhs T, Toumbourou J. Interventions for reducing alcohol supply, alcohol demand and alcohol-related harm: Final Report. Canberra: National Drug Law Enforcement Research Fund, 2015.

34. Martineau F, Tyner E, Lorenc T, et al. Population-level interventions to reduce alcohol-related harm: an overview of systematic reviews. Prev Med 2013:57:278-96.

35. Kingsland $\mathrm{M}$, Wolfenden L, Rowland BC, et al. Alcohol consumption and sport: a cross-sectional study of alcohol management practices associated with at-risk alcohol consumption at community football clubs. BMC Public Health 2013;13:762.

36. Rowland B, Tindall J, Wolfenden L, et al. Alcohol management practices in community football clubs: association with risky drinking at the club and overall hazardous alcohol consumption. Drug Alcohol Rev 2015;34:438-46.

37. Dietze PM, Fitzgerald JL, Jenkinson RA. Drinking by professional Australian Football League (AFL) players: prevalence and correlates of risk. Med J Aust 2008;189:479-83.

38. Aneni EC, Roberson LL, Maziak W, et al. A systematic review of internet-based worksite wellness approaches for cardiovascular disease risk management: outcomes, challenges \& opportunities. PLoS One 2014;9:e83594.
39. Luke DA, Calhoun A, Robichaux CB, et al. The program sustainability assessment tool: a new instrument for public health programs. Prev Chronic Dis 2014;11:130184.

40. Oinas-Kukkonen $\mathrm{H}$, Harjumaa M. Persuasive systems design: Key issues, process model, and system features. Communications of the Association for Information Systems: University of Oulu, 2009:24.

41. Wiltsey Stirman S, Kimberly J, Cook N, et al. The sustainability of new programs and innovations: a review of the empirical literature and recommendations for future research. Implement Sci 2012;7:17.

42. Rabin BA, Glasgow RE, Kerner JF, et al. Dissemination and implementation research on community-based cancer prevention: a systematic review. Am J Prev Med 2010;38:443-56.

43. Crisp BR, Swerissen H. Critical processes for creating healthpromoting sporting environments in Australia. Health Promot Int 2003:18:145-52.

44. Duff C, Munro G, Geoffrey M. Preventing alcohol-related problems in community sports clubs: the good sports program. Subst Use Misuse 2007;42:1991-2001.

45. Eime RM, Payne WR, Harvey JT. Making sporting clubs healthy and welcoming environments: a strategy to increase participation. $J \mathrm{Sci}$ Med Sport 2008;11:146-54.

46. Dobbinson SJ, Hayman JA, Livingston PM. Prevalence of health promotion policies in sports clubs in Victoria, Australia. Health Promot Int 2006;21:121-9.

47. Wiggers J, Considine R, Hazell T, et al. Increasing the practice of health promotion initiatives by licensed premises. Health Educ Behav 2001;28:331-40.

48. Wallin E, Andreásson S. Can I have a beer, please? A study of alcohol service to young adults on licensed premises in Stockholm. Prev Sci 2004;5:221-9.

49. Greenfield TK. Ways of measuring drinking patterns and the difference they make: experience with graduated frequencies. $J$ Subst Abuse 2000;12:33-49.

50. National Health and Medical Research Council. Australian guidelines to reduce health risks from drinking alcohol. Canberra: Commonwealth of Australia, 2009.

51. Conigrave KM, Saunders JB, Reznik RB. Predictive capacity of the AUDIT questionnaire for alcohol-related harm. Addiction 1995; $90: 1479-85$

52. Bauman A, Nutbeam D. Evaluation in a nutshell: a practical guide to the evaluation of health promotion programs. 2nd edn: McGraw Hill, 2013.

53. Miller P, Palmer D, Droste N, et al. Dealing with Alcohol-related problems in the Night-Time Economy: A Study Protocol for Mapping trends in harm and stakeholder views surrounding local community level interventions. BMC Res Notes 2011;4:204.

54. Kingsland $\mathrm{M}$, Wolfenden $\mathrm{L}$, Rowland $\mathrm{BC}$, et al. A cluster randomised controlled trial of a comprehensive accreditation intervention to reduce alcohol consumption at community sports clubs: study protocol. BMJ Open 2011;1: bmjopen2011000328.

55. White IR, Horton NJ, Carpenter J, et al. Strategy for intention to treat analysis in randomised trials with missing outcome data. BMJ 2011;342:d40.

56. Snow P, Haines K, stockwell TR, et al; The Prevention of Substance Use, Risk and Harm in Australia: a review of the evidence. Canberra: Commonwealth of Australia, 2004.

57. Nelson TF, Xuan Z, Babor TF, et al. Efficacy and the strength of evidence of U.S. alcohol control policies. Am J Prev Med 2013;45:19-28.

58. Wagenaar AC, Salois MJ, Komro KA. Effects of beverage alcohol price and tax levels on drinking: a meta-analysis of 1003 estimates from 112 studies. Addiction 2009;104:179-90.

59. National Drug Research Institute. Restrictions on the sale and supply of alcohol: evidence and outcomes. Perth: National Drug Research Insitute, Curtin University of Technology, 2007.

60. Gallet CA. The demand for alcohol: a meta-analysis of elasticities. Aust J Agric Resour Econ 2007;51:121-35.

61. Kelly B, King L, Bauman AE, et al. Identifying important and feasible policies and actions for health at community sports clubs: a consensus-generating approach. J Sci Med Sport 2014;17:61-6.

62. O'Brien KS, Kypri K, Kypros K. Alcohol industry sponsorship and hazardous drinking among sportspeople. Addiction 2008;103:1961-6.

63. Grossbard J, Geisner IM, Neighbors C, et al. Are drinking games sports? college athlete participation in drinking games and alcoholrelated problems. J Stud Alcohol Drugs 2007;68:97-105. 